\title{
An Experimental Study on Unipolar Induction
}

\author{
K. Chen, X.-J. Li* AND Y.-X. Hui \\ Institute of Photonics and Photon-Technology, Northwest University, Xi'an 710069, China \\ (Received September 14, 2016)
}

\begin{abstract}
Unipolar induction phenomenon is a special kind of electromagnetic induction. There are two quite opposite theoretical explanations for this phenomenon, i.e., the $N$ theory and the $M$ theory. The research of unipolar induction has made significant progress, but there is no final conclusion by now. In this paper, an experiment of inversely rotating double Faraday disks and double magnets are designed, and the unipolar induction phenomenon is verified by means of theoretical calculation and experiment. Comparing and analyzing the theoretical calculation and experiment results, our experimental results support the $N$ theory, that is to say, our experiment shows that the magnetic field does not rotate when the magnet rotates.
\end{abstract}

DOI: 10.12693/APhysPolA.131.271

PACS/topics: 01.55.+b, 03.50.De, 06.30.Ka, 41.20.Gz

\section{Introduction}

Unipolar induction phenomenon is a special kind of electromagnetic induction. When an axially symmetric permanent magnet rotates around its symmetry axis, induction current will be generated in the whole circuit which is formed by the conductive surface of the rotating magnet or a metal disk rotating synchronously with the magnet together with the stationary external circuit. There are two theories to explain the phenomenon of unipolar induction in the scientific community. One is Faraday's $N$ theory, the other is Webb's $M$ theory. The $N$ theory believes that the magnetic field does not rotate along with the magnet, and the rotating magnet itself or the rotating metal disk cuts the magnetic field to generate the induction electromotive force. The $M$ theory believes that the magnetic field rotates along with the magnet, and the static external circuit cuts the magnetic field to generate the induction electromotive force. Both of these two theories can explain the experimental phenomenon well. But there is only one truth, then, which theory can tell the real mechanism of the unipolar induction phenomenon?

Many experiments were carried out in order to determine which theory is really correct. In the beginning of twentieth century, the open loop circuit experiments by Barnett, Kennard, Pegram, et al. [1-7] were most representative in these experiments. Kennard's experimental conclusion was accepted by many people. He advocated denying the $M$ theory. Barnett's experimental conclusion was opposite. He advocated not denying the $M$ theory. They two had repeatedly heated debate. In 1970s, charge model based on quantum mechanics and the spin point magnetic dipole was proposed by Jehle and Djurić, respectively. This involved the old and awkward $M$ and $N$ theoretical hypothesis once again [8-10]. However, the generalized homopolar machine model proposed by Djurić made the hypothesis of unipolar induction

*corresponding author; e-mail: lixiaojun@nwu.edu.cn back to the origin [11]. When the generalized homopolar machine model was used to explain the past unipolar induction experiments from the perspective of $M$ hypothesis and $N$ hypothesis respectively, it was found that either the $M$ hypothesis or the $N$ hypothesis can explain the experimental phenomena well. After this, a new experiment was designed by Bartlett et al. to test and verify the $M$ hypothesis and $N$ hypothesis [12]. After a study on the results of this new experiment, they believed that $N$ theory is correct. This experiment was generally considered as highly accurate by the scientific community. After this, Kelly carried out a more comprehensive discussion. He designed simple and visual experiments, and then obtained conclusion that the $M$ theory is correct [13-15]. In 2006, article of He [16] from Huazhong University of Science and Technology questioned the experiment of Bartlett. Experiments of Macleod in 2012 and Müller in 2014 advocated $N$ theory [17, 18]. The debate between $N$ theory and $M$ theory continues until now.

The research of unipolar induction has made significant progress, but there is no final conclusion by now. In this paper, an experiment system composed of a pair of the Faraday disks and a pair of magnets which are rotating inversely to each other is designed. With the help of this experiment, we attempt to test and verify the theoretical interpretation of the unipolar induction phenomenon.

\section{Rotation experiment and measurement of induction electromotive force}

In this experiment, we plan to use double Faraday disks and double permanent magnets, and let the two permanent magnets rotate in opposite direction around their common symmetry axis. If rotation of the magnet can affect state of the magnetic field, overall motion state of magnetic field of the two inversely rotating magnets will significantly different from that of a single magnet, and then electromagnetic induction of this double magnets system will differ from that of the traditional unipolar induction experiment system. This will provide a scientific evidence for verification of unipolar induction phenomenon. 


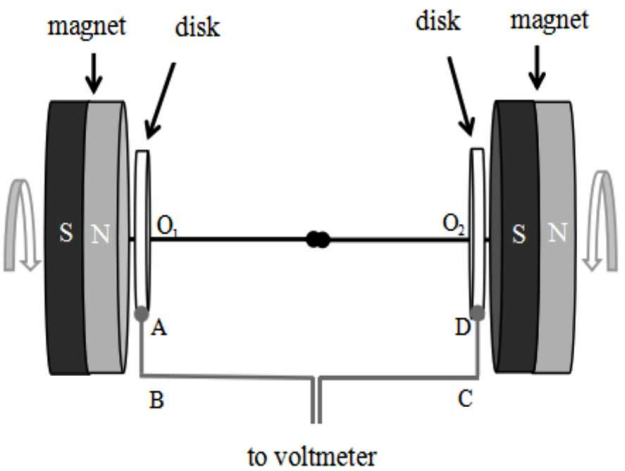

Fig. 1. Schematic diagram of the experiment device.

As shown in Fig. 1, opposite poles of the two circular magnets are arranged face to face. Between the two permanent magnets are two metal disks (the Faraday disks). The two disks are connected by a copper axis between them, the two disks and the external circuit are connected by electric brushes, and this forms a closed circuit loop. Two rotation systems are drived by two stepper motors and rotate in opposite direction around the same axis. Escapements are used to make the Faraday disks and permanent magnets independently rotating or stationary.

The two magnets used in the experiment are NdFeB permanent magnet rings, whose outer diameters are $70 \mathrm{~mm}$, inner diameters are $40 \mathrm{~mm}$, thicknesses are $6 \mathrm{~mm}$, and magnetization directions are at the thickness direction. The Faraday disks are aluminum disks whose diameters are $48 \mathrm{~mm}$. (Diameter of the disk is determined by computer simulation. Under present magnet and external circuit specifications, if the disks take this diameter, the induction electromotive force generated by rotating magnetic field and non-rotating magnetic field are significantly different from each other.) The distance between the two Faraday disks is $50 \mathrm{~mm}$. The gap between the disk and the permanent magnet surface is $3 \mathrm{~mm}$. Wire ABCD is the outer circuit, and it forms a rectangular loop together with the two Faraday disks and their common rotating axis. The whole rectangular loop is in the same plane. The distance between wire $\mathrm{BC}$ and the axis is $50 \mathrm{~mm}$. Length of wire $\mathrm{AB}$ and $\mathrm{CD}$ are $11 \mathrm{~mm}$ and length of wire $\mathrm{BC}$ is $50 \mathrm{~mm}$. The rotation experiments are carried out in two cases: (I) the two disks rotate and the permanent magnets remain stationary, the other is that the two disks rotate and (II) the magnet rotates synchronously with its corresponding disk (the disk on the same side of the magnet itself), respectively. The rotating direction of the two rotation systems is opposite and the angular velocity is equal to each other. The measurement results of induction electromotive forces in the two cases are shown in Table I.

It can be seen in Table I, within the error range, the induction electromotive force in the two cases is equal to each other. In fact, what are really useful for the verifi-
TABLE I

Measurement results of induction electromotive force $\varepsilon$ in the experiment.

\begin{tabular}{c|c|c}
\hline \hline \multirow{2}{*}{$\begin{array}{c}\omega / 2 \pi \\
{[1 / \mathrm{s}]}\end{array}$} & \multicolumn{2}{|c}{$\varepsilon[\mathrm{mV}]$} \\
\cline { 2 - 3 } & case I & case II \\
\hline 1 & 0.06 & 0.059 \\
\hline 2 & 0.13 & 0.128 \\
\hline 3 & 0.2 & 0.19 \\
\hline 4 & 0.27 & 0.26 \\
\hline 5 & 0.33 & 0.32
\end{tabular}

cation experiment are just the results when the disks and the permanent magnets rotate synchronously. The measurement result when the permanent magnets are static only acts as a reference so as to judge whether the experimental error is acceptable.

\section{Magnetic field measurement and theoretical calculation}

By measurement instrument of magnetic field, the magnetic field component in the circuit loop plane and perpendicular to the direction of the circuit is measured at points on the radius of the disks and the outer circuit. On the basis of the magnetic field distribution gained in the actual measurement, according to the electromagnetic induction law

$$
\varepsilon=\int_{\mathrm{L}}(\boldsymbol{\nu} \times \boldsymbol{B}) \cdot \mathrm{d} \boldsymbol{l},
$$

the induced electromotive force in the circuit loop can be calculated in several cases.

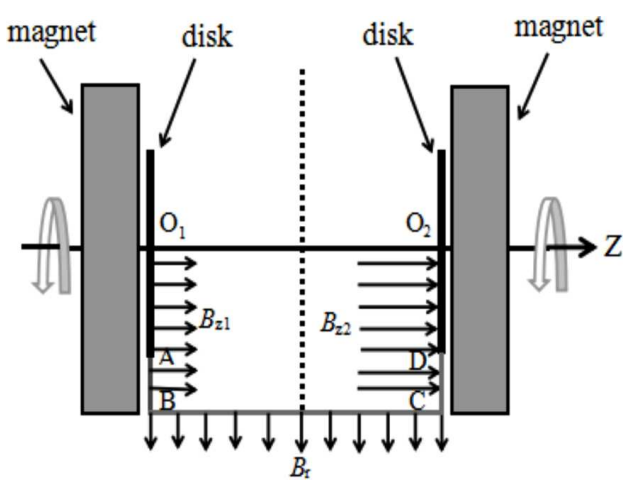

Fig. 2. Distribution of the magnetic field components of the magnet on the left which are in the loop plane and vertical to the circuit.

The magnetic field generated by $N$ pole of the magnet on the left side is shown in Fig. 2. The magnetic field component vertical to the disk at points on line segments $O_{1} B$ and $O_{2} C$ are $B_{z 1}(r)$ and $B_{z 2}(r)$, respectively $(r$ is radial distance), and their directions are along the positive $Z$ axis. The magnetic field component within the circuit loop plane and vertical to line $\mathrm{BC}$ at points on the longitudinal segment of the external circuit wire BC is $B_{r}(z)$ and the direction is outward along the radius. 
Now the two disks rotate in the opposite direction to each other at the same rotational speed, and the permanent magnet rotates synchronously with the corresponding disk.

The induction electromotive force generated by magnetic field of the left magnet can be calculated as below.

(1) Assume that the magnetic field does not rotate when the magnet is rotating. According to the law of electromagnetic induction, the induction electromotive force generated in each segment in the circuit loop follows respectively:

(i) $\quad \varepsilon_{\mathrm{AO} 1}=\int_{\mathrm{AO} 1} \nu_{\mathrm{AO} 1}(r) B_{z 1}(r) \mathrm{d} l=\int_{0}^{R} r \omega B_{z 1}(r) \mathrm{d} r$, where $\nu_{\mathrm{AO} 1}=r \omega$ is the speed of the disk cutting the magnetic field, $\omega$ is angular velocity of the disk, and $R$ is radius of the disk.

(ii) Because the external circuit remains stationary, it does not cut the magnetic field, therefore, $\varepsilon_{\mathrm{AB}}=\varepsilon_{\mathrm{BC}}=\varepsilon_{\mathrm{CD}}=0$.

(iii) $\quad \varepsilon_{\mathrm{DO} 2}=\int_{\mathrm{DO} 2} \nu_{\mathrm{DO} 2}(r) B_{z 2}(r) \mathrm{d} l=\int_{0}^{R} r \omega B_{z 2}(r) \mathrm{d} r$, where $\nu_{\mathrm{DO} 2}=r \omega$ is the speed of the disk cutting the magnetic field.

(2) Assume that the magnetic field rotates synchronously with the permanent magnet. The induction electromotive force generated in each segment in the circuit loop follows respectively:

(i) Because the left disk is synchronously rotating with the magnetic field, it does not cut the magnetic field, there is no induction electromotive force generated, so $\varepsilon_{\mathrm{AO} 1}=0$

(ii) $\quad \varepsilon_{\mathrm{AB}}=\int_{\mathrm{AB}} \nu_{\mathrm{AB}}(r) B_{z 1}(r) \mathrm{d} l=\int_{R}^{R_{0}} r \omega B_{z 1}(r) \mathrm{d} r$, where $\nu_{\mathrm{AB}}=r \omega$ is the speed of wire $\mathrm{AB}$ cutting the magnetic field, and $R_{0}$ is radial distance of point $B$.

(iii) $\quad \varepsilon_{\mathrm{BC}}=\int_{\mathrm{BC}} \nu_{\mathrm{BC}}(z) B_{r}(z) \mathrm{d} l=\int_{0}^{L} R_{0} \omega B_{r}(z) \mathrm{d} z$, where $\nu_{\mathrm{BC}}=R_{0} \omega$ is the speed of wire $\mathrm{BC}$ cutting the magnetic field, $R_{0}$ is the distance between wire $\mathrm{BC}$ and the axis, and $L$ is length of wire BC.

(iv) $\quad \varepsilon_{\mathrm{CD}}=\int_{\mathrm{CD}} \nu_{\mathrm{CD}}(r) B_{z 2}(r) \mathrm{d} l=\int_{R}^{R_{0}} r \omega B_{z 2}(r) \mathrm{d} r$, where $\nu_{\mathrm{CD}}=r \omega$ is the speed of wire $C D$ cutting the magnetic field.

(v) $\quad \varepsilon_{\mathrm{DO} 2}=\int_{\mathrm{DO} 2} \nu_{\mathrm{DO} 2}(r) B_{z 2}(r) \mathrm{d} l=\int_{0}^{R} 2 r \omega B_{z 2}(r) \mathrm{d} r$. Here because rotation direction of the right disk is opposite to that of the left magnet, the velocity of the right disk cutting the magnetic field of the left magnet is $\nu_{\mathrm{DO} 2}=2 r \omega$.

The magnetic field generated by $\mathrm{S}$ pole of the magnet on the right side is shown in Fig. 3. The magnetic field component vertical to the disk at points on line segments $O_{1} B$ and $O_{2} C$ are $B_{z 3}(r)$ and $B_{z 4}(r)$, respectively, and their directions are along the positive $Z$ axis. The magnetic field component within the circuit loop plane and vertical to $B C$ at points on wire $B C$ is $B_{r}(z)$ and the direction is inward along the radius. The two disks rotate in opposite direction to each other at the same rotational speed and the permanent magnet rotates synchronously with the corresponding disk.

The induction electromotive force generated by magnetic field of the right magnet can be calculated as below.

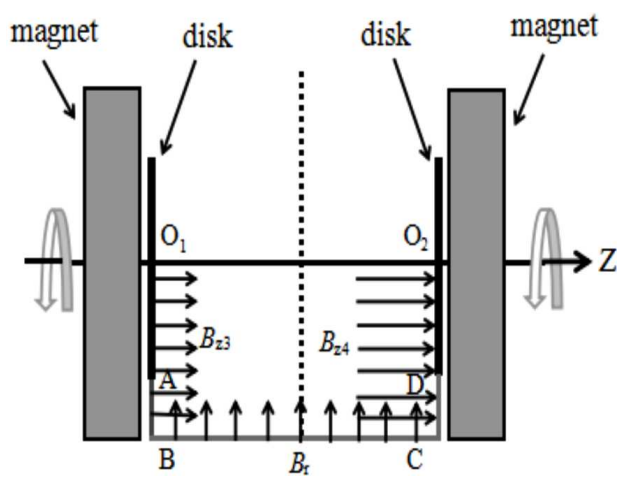

Fig. 3. Distribution of the magnetic field component of the magnet on the right which are in the loop plane and vertical to the circuit.

(1) Assume that the magnetic field does not rotate with the magnet. According to the law of electromagnetic induction, the induced electromotive force generated in each segment of the circuit loop follows respectively:

(i) $\quad \varepsilon_{\mathrm{AO} 1}^{\prime}=\int_{\mathrm{AO} 1} \nu_{\mathrm{AO} 1}(r) B_{z 3}(r) \mathrm{d} l=\int_{0}^{R} r \omega B_{z 3}(r) \mathrm{d} r$, where $\nu_{\mathrm{AO} 1}=r \omega$ is the speed of the disk cutting magnetic field.

(ii) Because the external circuit remains stationary, the external circuit does not cut the magnetic field, therefore, $\varepsilon_{\mathrm{AB}}^{\prime}=\varepsilon_{\mathrm{BC}}^{\prime}=\varepsilon_{\mathrm{CD}}^{\prime}=0$.

(iii) $\varepsilon_{\mathrm{DO} 2}^{\prime}=\int_{\mathrm{DO} 2} \nu_{\mathrm{DO} 2}(r) B_{z 4}(r) \mathrm{d} l=\int_{0}^{R} r \omega B_{z 4}(r) \mathrm{d} r$, where $\nu_{\mathrm{DO} 2}=r \omega$ is the speed of the disk cutting magnetic field.

(2) Assume that the magnetic field rotates synchronously with the magnet, the induction electromotive force generated in each segment of the circuit loop follows respectively:

(i) $\quad \varepsilon_{\mathrm{AO} 1}^{\prime}=\int_{\mathrm{AO} 1} \nu_{\mathrm{AO} 1}(r) B_{z 3}(r) \mathrm{d} l=\int_{0}^{R} 2 r \omega B_{z 3}(r) \mathrm{d} r$. Here, because rotation direction of the left disk is opposite to that of the right magnet, the velocity of the left disk cutting the magnetic field is $\nu_{\mathrm{AO} 1}=2 r \omega$.

(ii) $\quad \varepsilon_{\mathrm{AB}}^{\prime}=\int_{\mathrm{AB}} \nu_{\mathrm{AB}}(r) B_{z 3}(r) \mathrm{d} l=\int_{R}^{R_{0}} r \omega B_{z 3}(r) \mathrm{d} r$, where $\nu_{\mathrm{AB}}=r \omega$ is the speed of wire $\mathrm{AB}$ cutting the magnetic field.

(iii) $\quad \varepsilon_{\mathrm{BC}}^{\prime}=\int_{\mathrm{BC}} \nu_{\mathrm{BC}}(z) B_{r}(z) \mathrm{d} l=\int_{0}^{L} R_{0} \omega B_{r}(z) \mathrm{d} z$, where $\nu_{\mathrm{BC}}=R_{0} \omega$ is the speed of wire $\mathrm{BC}$ cutting the magnetic field.

(iv) $\quad \varepsilon_{\mathrm{CD}}^{\prime}=\int_{\mathrm{CD}} \nu_{\mathrm{CD}}(r) B_{z 4}(r) \mathrm{d} l=\int_{R}^{R_{0}} r \omega B_{z 4}(r) \mathrm{d} r$, where $\nu_{\mathrm{CD}}=r \omega$ is the speed of wire CD cutting the magnetic field.

(v) Because the left disk is synchronously rotating with the magnetic field, it does not cut the magnetic field, there is no induction electromotive force generated, so $\varepsilon_{\mathrm{DO} 2}^{\prime}=0$.

Combining the induction electromotive force in Fig. 2 and Fig. 3, when the two disks and the magnets are rotating synchronously, there will be:

(1) Assume that the magnetic field does not rotate with the magnet, the induction electromotive force generated by all of the two magnets is

$$
\varepsilon_{N}=\varepsilon_{\mathrm{AO} 1}+\varepsilon_{\mathrm{AO} 1}^{\prime}+\varepsilon_{\mathrm{DO} 2}+\varepsilon_{\mathrm{DO} 2}^{\prime} .
$$


(2) Assume that the magnetic field rotates synchronously with the magnet, the induction electromotive force generated by all of the two magnets is

$$
\begin{aligned}
& \varepsilon_{M}=\varepsilon_{\mathrm{BC}}+\varepsilon_{\mathrm{CD}}+\varepsilon_{\mathrm{DO} 2}-\varepsilon_{\mathrm{AB}}+\varepsilon_{\mathrm{AO} 1}^{\prime}+\varepsilon_{\mathrm{AB}}^{\prime} \\
& \quad+\varepsilon_{\mathrm{BC}}^{\prime}-\varepsilon_{\mathrm{CD}}^{\prime} .
\end{aligned}
$$

Finally, according to the magnetic field measurement results, the specific values of induction electromotive force are calculated and shown in Table II.

\section{TABLE II}

Theoretical calculation of induction electromotive force $\varepsilon$.

\begin{tabular}{c|c|c}
\hline \hline \multirow{2}{*}{$\omega / 2 \pi$} & \multicolumn{2}{|c}{$\varepsilon[\mathrm{mV}]$} \\
\cline { 2 - 3 }$[1 / \mathrm{s}]$ & $\varepsilon_{N}$ & $\varepsilon_{M}$ \\
\hline 1 & 0.063 & 0.015 \\
2 & 0.126 & 0.029 \\
3 & 0.189 & 0.044 \\
4 & 0.252 & 0.058 \\
5 & 0.315 & 0.073
\end{tabular}

It can be seen in Table II that the induction electromotive force in the case when assuming the magnetic field does not rotate with the magnet is obviously different from the case when assuming the magnetic field rotates synchronously with the magnet. This provides the possibility to judge whether the magnetic field is rotating in the experiment.

Comparing Tables I and II, it can be found that the induction electromotive force measured in rotating magnet experiments are quite close to the theoretical calculation results in the case when assuming the magnetic field is stationary. This shows that the magnetic field does not rotate with the magnet when the permanent magnet rotates.

\section{Summary}

In this paper, an experiment of inversely rotating double Faraday disks and double magnets are designed.
The unipolar induction phenomenon is studied by means of theoretical calculation and experiment. Comparing and analyzing the theoretical calculation and experiment results, theoretical interpretation of the unipolar induction is verified and determined. Our experimental results support the $N$ theory, that is to say, our experiment shows that the magnetic field does not rotate when the magnet rotates.

\section{References}

[1] G.B. Pegram, Phys. Rev. 10, 591 (1917).

[2] S.J. Barnett, Phys. Rev. 35, 323 (1912).

[3] S.J. Barnett, Phys. Rev. 2, 323 (1913).

[4] S.J. Barnett, Phys. Rev. 12, 95 (1918).

[5] E.H. Kennard, Philos. Mag. 23, 937 (1912).

[6] E.H. Kennard, Phys. Rev. 1, 355 (1913).

[7] E.H. Kennard, Philos. Mag. 33, 179 (1917).

[8] H. Jehle, Phys. Rev. D 3, 306 (1971).

[9] H. Jehle, Phys. Rev. D 11, 2147 (1975).

[10] H. Jehle, Phys. Rev. D 6, 441 (1972).

[11] J. Djurić, J. Appl. Phys. 46, 679 (1975).

[12] D.F. Barlett, J. Monroy, J. Reeves, Phys. Rev. D 16, 3459 (1977).

[13] A.G. Kelly, Faraday's Final Riddle: Does the Field Rotate with a Magnet, Inst. Engrs. Ireland. Monograph No. 6, Ireland 1998.

[14] A.G. Kelly, Phys. Essays 12, 372 (1999).

[15] A.G. Kelly, Ann. Fondat. Louis de Broglie 29, 119 (2004).

[16] Y. He, M.Sc. Thesis, Huazhong University of Science and Technology, 2016 (in Chinese).

[17] N. Macleod, Phys. Essays 25, 524 (2012).

[18] F.J. Müller, IEEE Trans. Magn. 50, 1 (2014). 